\title{
DISTRIBUTIONS OF THE SOURCES OF THE MAGNETIC FIELD DURING THE DOUBLE MAGNETIC CYCLE
}

\author{
ELENA E. BENEVOLENSKAYA \\ Pulkovo Observatory \\ St. Petersburg, 196140, Russia
}

The longitudinal and latitudinal distributions of the solar magnetic field have been investigated by many authors see e.g. (Bumba and Howard 1969; Gaizauskas et. al. 1983). The main longitudinal structures such as active longitudes, sector boundaries in the solar wind, and coronal holes appear to be the consequence of non-symmetric magnetic modes (Stix 1971; Ivanova and Ruzmaikin 1977) and can be explained within the framework of dynamo models. According to these models, solar magnetic fields are generated by helicity and differential rotation in the solar convection zone (Parker 1979) and the linear dynamo process may generate a carrier frequency $\omega_{c}\left(\omega_{c}=2 \pi / T, T=22 \mathrm{yr}\right)$.

The 22-yr cycle of solar activity is a magnetic cycle that manifests itself as a change of polarity of the sunspots. In parallel with this, the poloidal magnetic field, or background field ( $B_{r}$-component), shows a 22 -yr periodicity (Howard and LaBonte 1981).

According to the studies (Stenflo 1994) the low-latitude and polar fields clearly show the 22-year periodicity too and there is an indication for lowamplitude power at higher frequencies, corresponding to periods of about $2 \mathrm{yr}$. In non-linear theory stable frequencies with a period shorter than that of the cycle period may occur (Hoyng 1990). All above mentioned allow to assume that cycle consists of two component: high frequency (Hale cycle) and low frequency (biennial cycle). The purpose of our investigation is to find longitudinal structure of the solar magnetic cycle.

Line-of-sight $\left(B_{\|}\right)$magnetograph data observed by the Wilcox Solar Observatory at Stanford are used in the present investigation. Synoptic observations of the Sun's photospheric magnetic field were obtained covering the interval from 1976 to 1996, spanning cycles 21 and 22 .

In the analysis, the first harmonic $\left(f_{1}\right)$ corresponds to $T_{1}=256$ Carrington rotations (being, approximately, $20 \mathrm{yr}$ ), and the tenth harmonic $\left(f_{10}\right)$ corresponds to $T_{10}=T_{1} / 10$ (being, approximately, $2 \mathrm{yr}$ ). The results are obtained as a power spectrum $P_{y y}(\mu, f)(\mu=\cos \theta)$ for each longitude $l_{i}$. Finally, the 
power spectra $P_{y y}(\mu, f)$ were averaged over latitude, separately, for northern and southern hemispheres.

This investigation has revealed that the low-frequency $\left(f_{1}\right)$ and high- frequency $\left(f_{10}\right)$ components of longitudinal distribution are distributed nonuniformly on the solar surface and rotate approximately at the same rate. In particular, both the low-frequency $\left(f_{1}\right)$ and high-frequency $\left(f_{10}\right)$ components reach their maximum values in different longitudinal zones that are separated by $20^{\circ}$.

A natural question is whether differences between the latitudinal distribution of the low-frequency $f_{1}$ component and high-frequency $f_{10}$ component take place for a given longitude.

The first harmonic $f_{1}$, having a period $T_{1}=20 \mathrm{yr}$, appears dominant and has a maximum at latitudes greater than $40^{\circ}$ in both hemispheres, as does the $f_{10}$ component with $T_{10}=2.0 \mathrm{yr}$. These results coincide with those found in the distribution of high-frequency and low-frequency components of the magnetic field for the axisymmetrical case (Benevolenskaya 1994, 1995). Thus, it may be conceivable that these distributions correspond to the reality to the physical picture of a double magnetic cycle of which both components reach their maximum values at high latitude.

Therefore, we conclude that the poloidal magnetic field of the solar activity cycle consists of both axisymmetrical and non-axisymmetrical modes: a highfrequency (biennial) component and a low-frequency (Hale) component.

\section{Acknowledgements}

I am grateful to Dr. J. Todd Hoeksema (Stanford University) for providing the Wilcox Solar Observatory line-of-sight magnetograph data, Prof. Toshio Fukushima for a possibility to attend the GA IAU (LOC IAU grant) and Prof. Franz-L. Deubner for useful comments.

\section{References}

Benevolenskaya, E. E. 1994, Astron. Lett., 20, 468

Benevolenskaya, E. E. 1995, Solar Phys., 161, 1

Bumba, V. and Howard, R. 1969, Solar Phys., 7, 28

Gaizauskas, V., Harvey, K. L., Harvey, J. W. and Zwaan, C. 1983, Astrophys. J., 265, 1056

Hoyng, P. 1990, Solar Photosphere: Structure, Convection and Magnetic Fields, Stenflo, J. O., IAU Symp. 138, 359

Howard, R. and LaBonte, B. J. 1981, Solar Phys., 74, 131

Ivanova, T. S. and Ruzmaikin, A. A. 1977, Astron. Zh., 54, 846

Parker, E. N. 1979, Cosmical Magnetic Fields, Oxford University Press

Stenflo, J. O. 1994, Solar Surface Magnetism, Rutten, R. J. and Schrijver C. J., NATO Advanced Research Workshop, Kluwer

Stix, M., 1971, Astron. Astrophys., 13, 203 